\title{
Percutaneous transvenous intracardiac ultrasound imaging in dogs: a new approach to monitor left ventricular function
}

\author{
L Jiang, N J Weissman, J L Guerrero, J He, A E Weyman, R A Levine, M H Picard
}

\begin{abstract}
Objective-To evaluate the feasibility and ability of percutaneous transvenous intracardiac echocardiography (ICE) to image the left ventricle (LV) and monitor its function from the right ventricular (RV) cavity.
\end{abstract}

Methods-A $10 \mathrm{MHz}$ catheter was advanced into the $R V$ from the jugular vein and positioned along the septum at the $L V$ papillary muscle level in five dogs. The catheter was manipulated until a stable catheter position along the septum, which provided on-axis images of the $L V$, was obtained. Different states of $\mathrm{LV}$ size and systolic function $(n=80)$ were created with dobutamine or esmolol, both in the presence and absence of coronary stenoses. LV stroke area $\left(\mathrm{cm}^{2}\right)$ obtained by ICE was measured at the mid-ventricular level and compared with stroke volume $\left(\mathrm{cm}^{3}\right)$ obtained simultaneously with a transaortic flow probe. LV end diastolic, end systolic, and stroke areas obtained by ICE were also compared with those obtained by short-axis epicardial echocardiography.

Results-In $96 \%$ of the stages, short axis images of the LV could be obtained and measured by ICE. LV end diastolic, end systolic, and stroke areas measured by ICE were not significantly different from epicardial echocardiographic values. Stroke area correlated with stroke volume in each dog (mean correlation coefficient $0.79\left(\right.$ SEE 0.19) $\left.\mathrm{cm}^{2}\right)(P<0.001)$.

Conclusions-Percutaneous intracardiac ultrasound imaging allows monitoring of $L V$ function from the RV with an accuracy comparable to a short-axis epicardial echocardiogram. The present device can be used in closed chest experimental studies. With the development of lower frequency devices, this technique may be valuable for continuous monitoring of $\mathrm{LV}$ function in patients in the intensive care unit or operating room.

(Heart 1996;76:442-448)

Keywords: intracardiac echocardiography; left ventricular function; monitoring; systolic function
Catheter-based ultrasound transducers can now be positioned directly into cardiac chambers to give a clear delineation of the endocardial borders and other cardiac structures. This approach can give accurate measurements of ventricular volume $^{1-3}$ and valve orifice. ${ }^{4}$ Initially, these intracardiac echocardiography (ICE) images were obtained with high frequency $(20 \mathrm{MHz})$ intravascular ultrasound catheters with a limited depth of field because of poor penetration of the sound beam. This required the catheter to be very close to the structure of interest. Thus to image and measure left ventricular structure and function, the catheter had to be placed within the left ventricular cavity. With the introduction of lower frequency $(10 \mathrm{MHz})$ ICE transducers, the resolution of structures at greater depths improved, resulting in the potential of imaging the left ventricle from outside the chamber. ${ }^{56}$ The purpose of this study was to evaluate the feasibility of percutaneous transvenous intracardiac ultrasound imaging of the left ventricle from a position within the right ventricle and to determine the ability of this approach to monitor left ventricular systolic function in vivo.

\section{Methods}

EXPERIMENTAL MODEL

Five mongrel dogs with a mean (SD) weight of $28.4(7 \cdot 4) \mathrm{kg}$ (range $17-38 \mathrm{~kg}$ ) were anaesthetised with pentobarbitone sodium $(10 \mathrm{mg} / \mathrm{kg}$ intravenously) and mechanically ventilated. Supplemental doses were given as needed during the subsequent procedures to maintain an adequate level of anaesthesia. The concentration of inspired oxygen and ventilation rate were adjusted to keep blood gases within the physiological range. A midline thoracotomy was performed, the pericardium incised, and the heart suspended in a pericardial cradle. An ultrasound flow probe (Transonic Systems, Ithaca, NY) was positioned on the ascending aorta for continuous measurement of stroke volume.

INTRACARDIAC ULTRASOUND

A commercially available $10 \mathrm{MHz}$ transducer mounted on a $10 \mathrm{~F}$ catheter (Cardiovascular Imaging Systems, Sunnyvale, CA) was used for all intracardiac imaging. The catheter has a 

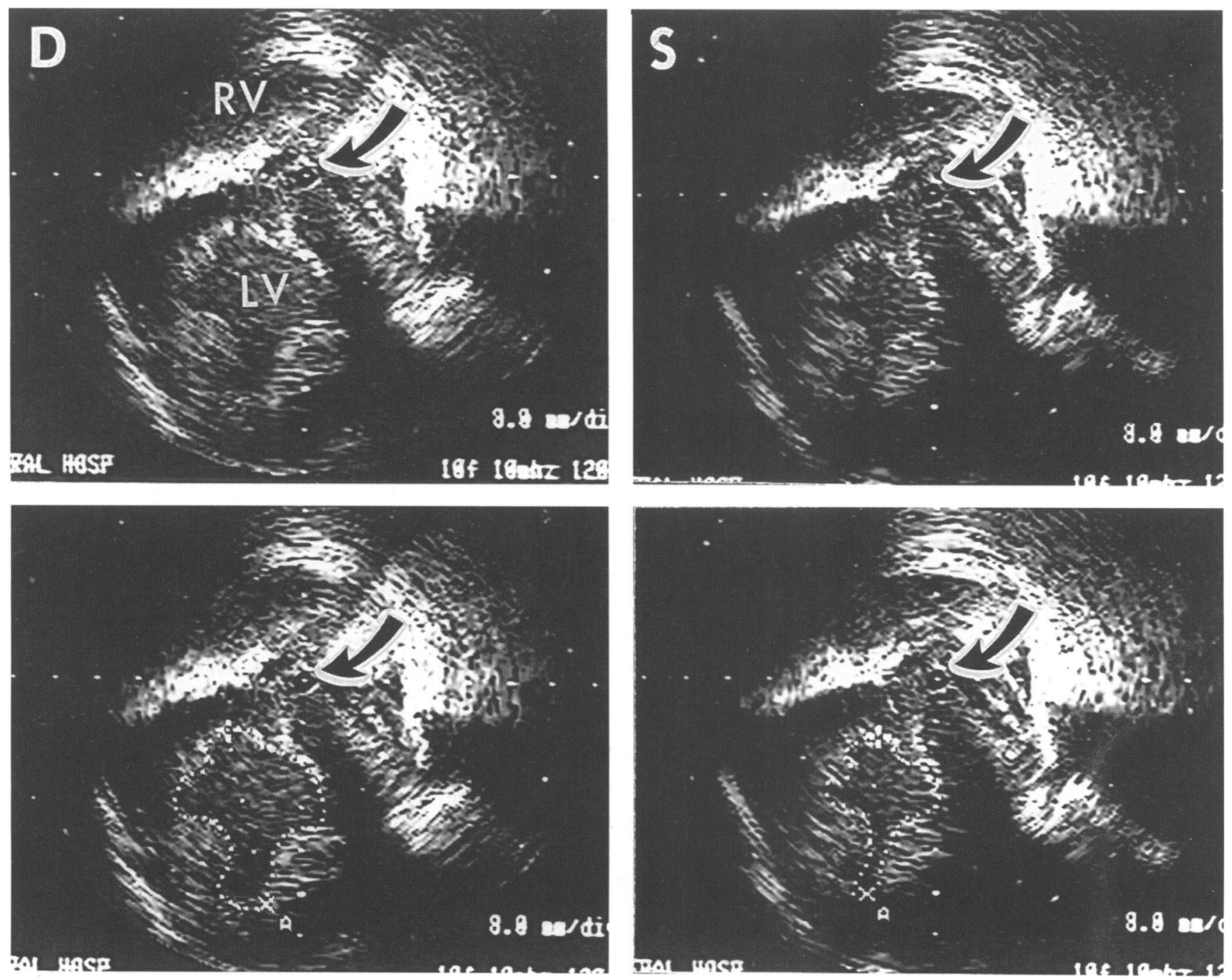

Figure 1 Examples of intracardiac echocardiographic cross sectional images of the LV from the $R V$ in diastole (upper left), and in systole (upper right). The bottom panels display tracings of endocardial borders to measure area. Arrow indicates catheter. $L V$, left ventricle; $R V$, right ventricle; $S$, systole; $D$, diastole.

fixed transducer at its tip with a mirror rotating at $1800 \mathrm{rpm}$ to produce a tomographic image. The image has an axial resolution of $0.30 \mathrm{~mm}$, lateral resolution of $0.37 \mathrm{~mm}$, and depth of penetration of about $6 \mathrm{~cm}$. The ICE catheter was advanced through a $10 \mathrm{~F}$ introducer sheath in the right internal jugular vein

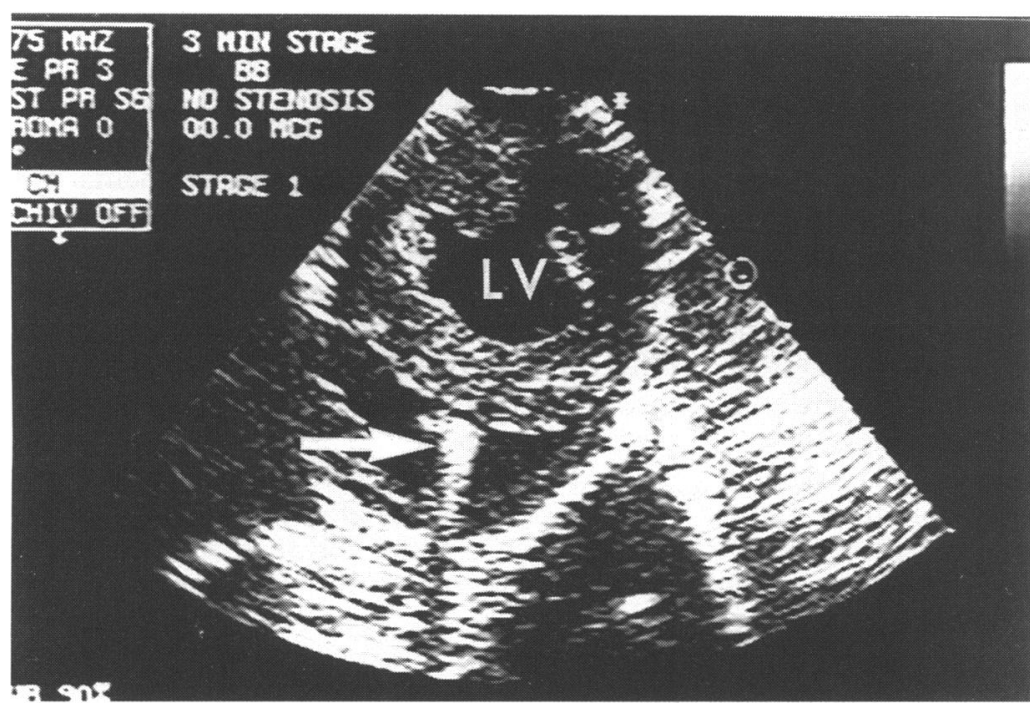

Figure 2 Example of epicardial echocardiographic short axis image of the $L V$, with an ICUS catheter (arrow) positioned along the interventricular septum. LV, left ventricle. into the right ventricle and positioned along the interventricular septum. The position of the catheter was manipulated to obtain cross sectional images at the mid left ventricle in which both papillary muscles were imaged symmetrically (fig 1). Once an optimal catheter position was obtained, there was no further manipulation of the ICE catheter for the duration of the experiment. Intracardiac echocardiographic images were continuously recorded on 0.5 inch videotape.

\section{EPICARDIAL ECHOCARDIOGRAPHY}

Epicardial echocardiographic imaging of the open-chest preparation was performed to identify the occurrence of wall motion abnormalities. This was performed with the aid of a water bath for acoustical coupling and transducer standoff. Short axis images of the left ventricle at the papillary muscle level were recorded using an Interspec Apogee CX200 echocardiograph (Interspec ATL, Ambler, PA) operating at $3.5 \mathrm{MHz}$ (fig 2). In order to confirm the accuracy of ICE measurements of ventricular area, measurements of $\mathrm{LV}$ diastolic, systolic, and stroke area (defined below) obtained nearly simultaneously by epicardial echocardiography were performed for one dog. 
Figure 3 Correlations between ICUS and epicardial echo in the measurement of $L V$ diastolic (top), systolic (middle), and stroke area (bottom) for one dog.The line of identity is shown.
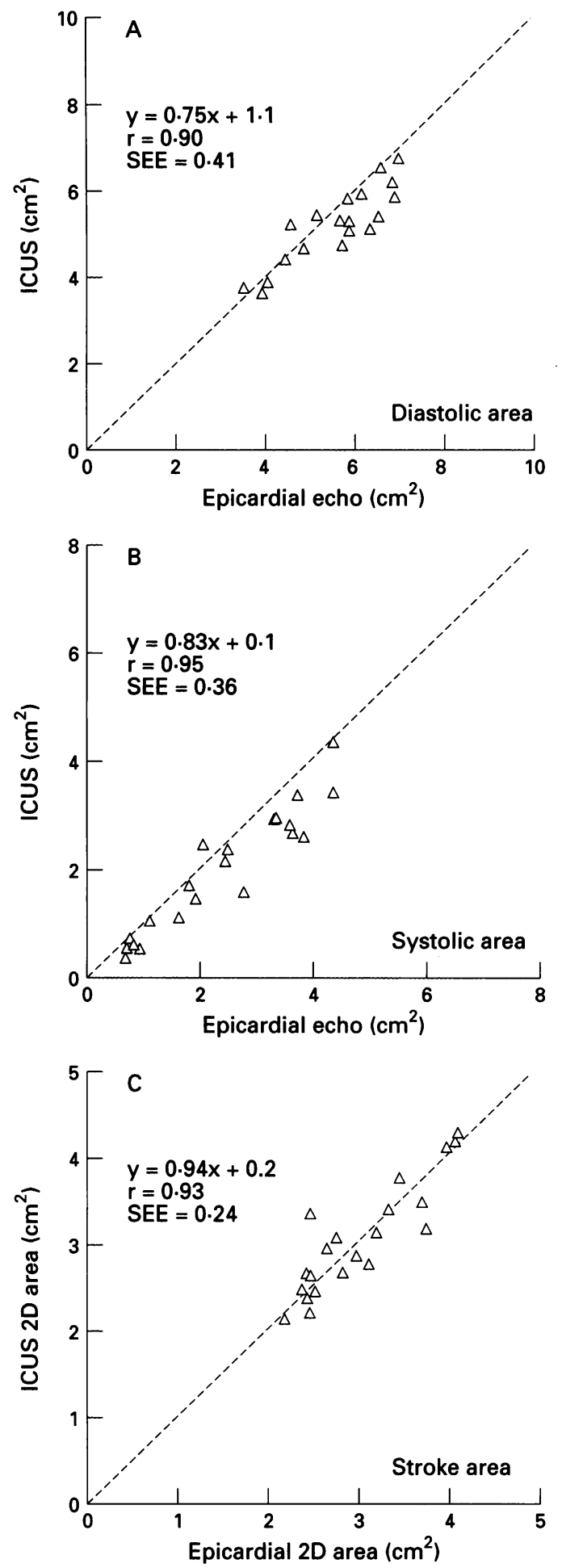

EXPERIMENTAL PROTOCOL

To test the imaging capabilities of the right ventricular intravascular ultrasound catheter to image left ventricles of different sizes and shapes and with different degrees of systolic function, a variety of manoeuvres were performed to alter the haemodynamic state and left ventricular wall motion. Contractility was augmented and preload reduced with dobutamine (5 to $40 \mu \mathrm{g} / \mathrm{kg} / \mathrm{min}$ ). Esmolol $(100 \mu \mathrm{g} / \mathrm{kg} / \mathrm{min})$ was administered to reduce global contractility and increase heart size. Varying degrees of coronary stenoses were created in the proximal left anterior descending artery by a pneumatic occluder (Biomedical products, Silver Springs, MD). With this occluder the severity of the stenosis could be modified and this was quantified by the degree to which the hyperaemic response was blunted. Degrees of stenosis were selected which blunted coronary flow reserve without significantly decreasing baseline coronary flow. The dobutamine infusion in the setting of the coronary stenoses led to varying degrees of ischaemia and wall motion abnormality. By using different combinations of these three interventions, a total of 83 different haemodynamic states were created in five dogs. During each stage, intracardiac cross sectional echocardiographic images were recorded simultaneously with the recording of stroke volume from the transaortic flow probe.

This experiment conformed to the guiding principles of the American Physiological Association and was approved by the subcommittee on research animal care at the Massachusetts General Hospital.

DATA ANALYSIS

Cross sectional intracardiac and epicardial echo images of the left ventricle were reviewed off-line in a random order independent of knowledge of stroke volume, coronary stenosis status, dobutamine dose, or esmolol dose. The presence or absence of regional wall motion abnormalities were independently noted for the epicardial and intracardiac images by an experienced echocardiographer (LJ). Because earlier studies showed a linear relation between stroke volume and area, ${ }^{78}$ these two indices were calculated and compared. End diastolic images were defined as those with the largest LV cross sectional area and end systolic images were defined as those with the smallest LV cross sectional area. The endocardial borders of the mid-papillary level end diastolic and end systolic images for each stage were manually traced (fig 1 ). LV stroke area $\left(\mathrm{cm}^{2}\right)$ was calculated as the difference between diastolic and systolic areas.

STATISTICAL ANALYSIS

In each animal, LV stroke area measured by ICE was compared with LV stroke volume (from the transaortic flow probe) by linear regression analysis. Linear regression analysis was used to compare LV stroke area calculated from ICE with LV stroke area measured by epicardial echo in one animal. The mean difference between pairs of measurements was calculated according to the method described by Bland and Altman. ${ }^{9}$ Interobserver variability was expressed as the coefficient of variation, where the standard deviation of the differences between 36 ICE area measurements (18 end diastolic and 18 systolic areas) made by two investigators (LJ, $\mathrm{JH}$ ) was divided by the mean value measured. To test the intraobserver variability, the 36 ICE area measurements were repeated by the same observer (LJ) after a two week interval.

\section{Results}

The entire LV endocardial circumference throughout the cardiac cycle could be success- 

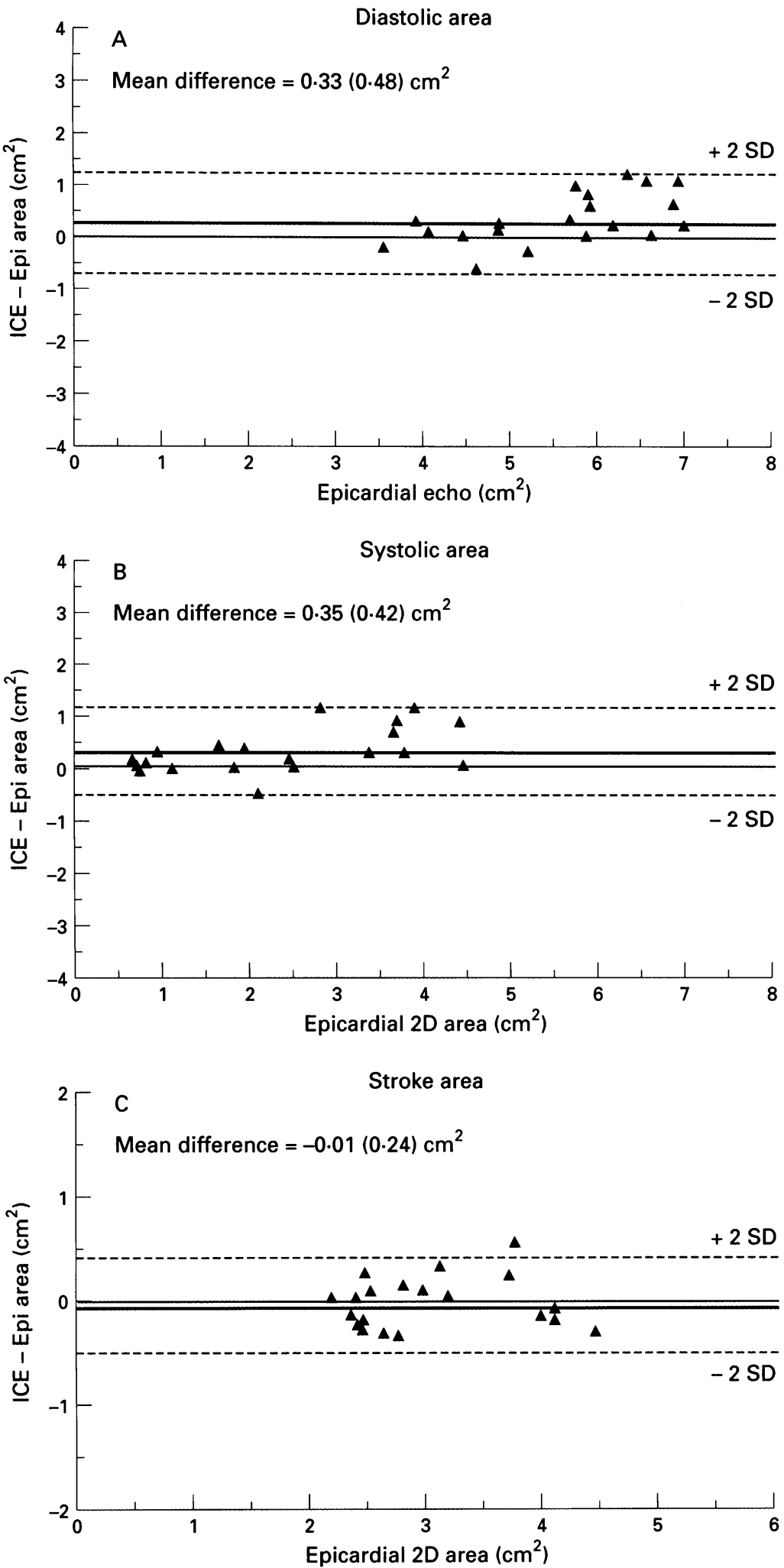

Figure 4 Bland-Altman analysis of LV diastolic area, systolic area, and stroke area for intracardiac and epicardial echocardiogram. ICE, intracardiac echocardiography; Epi, epicardial. the experiment, providing real-time monitoring of $\mathrm{LV}$ wall motion. Wall motion abnormalities $(n=26)$ were detected by ICE in all stages when they were present by epicardial echocardiography.

\section{AREAS: ICE VERSUS EPICARDIAL} ECHOCARDIOGRAPHY

End diastolic, end systolic, and stroke area measurements by the ICE and epicardial ultrasound correlated well (fig 3). The mean differences between left ventricular area measurements by the two techniques were: end diastolic area $0.33(0.48) \mathrm{cm}^{2}$, end systolic area $0.35(0.42) \mathrm{cm}^{2}$, and stroke area -0.01 $(0 \cdot 24) \mathrm{cm}^{2}$ (fig 4$)$. There was no statistical difference between the LV area measured by ICE and epicardial echocardiographic techniques.

\section{ICE STROKE AREA VERSUS FLOW PROBE STROKE VOLUME}

The relations between stroke area (ICE) and stroke volume (flow probe) for each dog are shown in fig 5. A linear relation was present with $\mathrm{r}$ values ranging from 0.72 to 0.87 (mean $\mathrm{r}=0.82(0.06) \mathrm{cm}^{3}$; mean standard error $=$ $\left.0.47(0 \cdot 16) \mathrm{cm}^{2}\right)$.

\section{REPRODUCIBILITY OF ICE MEASUREMENTS}

Interobserver variability for ICE area measurements was $5.5 \%$ and the intraobserver variability was $4 \cdot 6 \%$.

\section{Discussion}

The miniaturisation of ultrasound transducers sparked the development of both catheter based intravascular and intracardiac ultrasound imaging. These high resolution, real time images have been applied for the accurate evaluation of vascular structure and function. ${ }^{1011}$ However, with the standard high frequency transducers used for intravascular ultrasound, the poor resolution outside of a small depth of field has limited their widespread application to intracardiac ultrasound. Thus, monitoring of LV function would require the catheter to be positioned inside the LV in close proximity to all walls. ${ }^{1}$ This necessity for direct introduction into the $\mathrm{LV}$ makes the device impractical for monitoring $\mathrm{LV}$ function in most clinical settings. With the development of lower frequency transducers, resolution of structures at greater depths has been demonstrated. ${ }^{5}$ Our study adds to this observation by demonstrating that intracardiac ultrasound imaging of the LV obtained with a $10 \mathrm{MHz}$ transducer by percutaneous introduction of the catheter into the $R V$, provides adequate visualisation and measurement of the LV cavity in this canine model. Reproducible, serial LV cross sectional area measurements could be obtained by this ICE imaging approach and were found to be similar to those obtained by epicardial echocardiography. Furthermore, quantitative assessment of left ventricular systolic function was possible with these cross sectional images.

In this study a single cross sectional area change (stroke area) was measured and found 

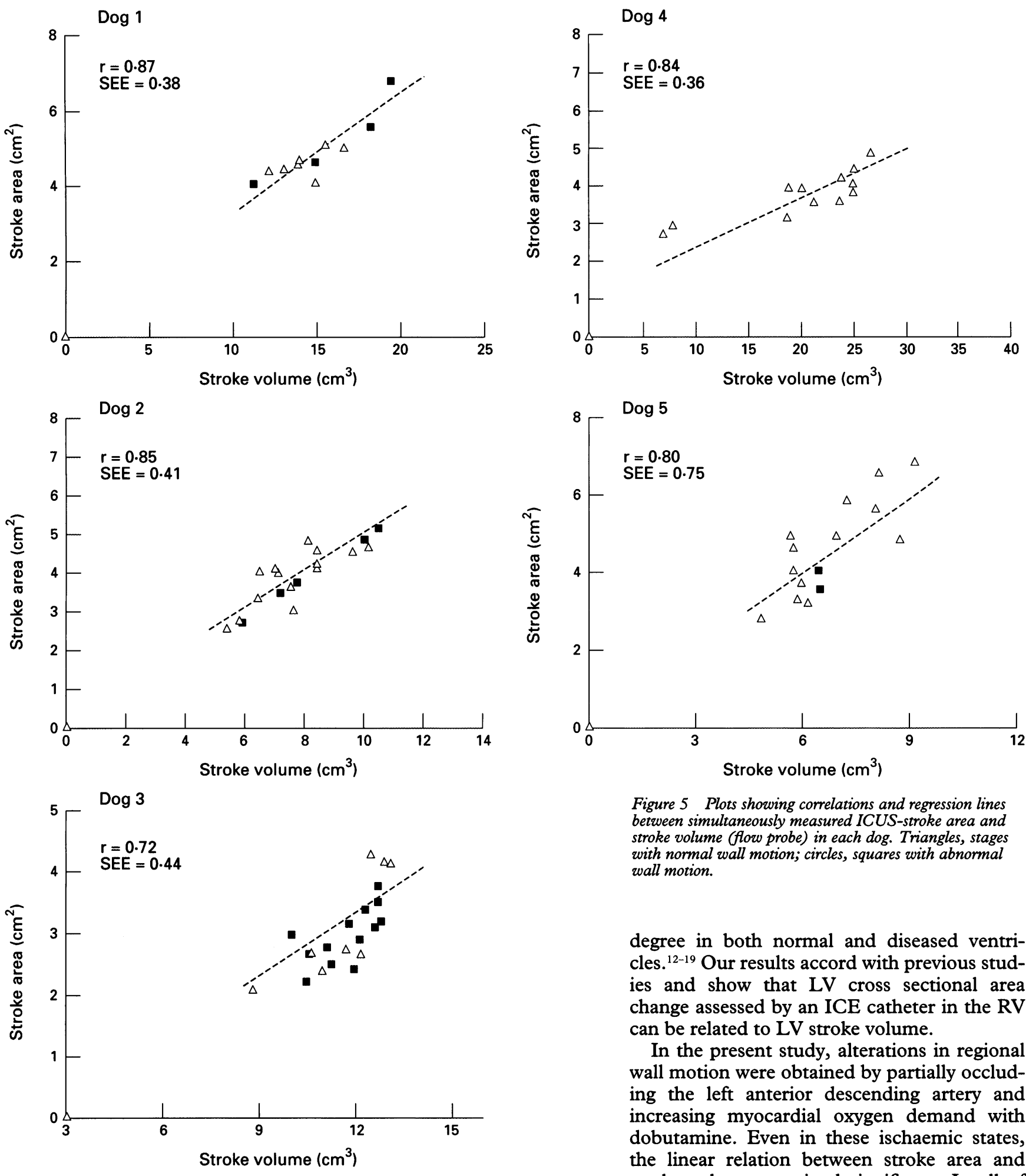

Figure 5 Plots showing correlations and regression lines between simultaneously measured ICUS-stroke area and stroke volume (flow probe) in each dog. Triangles, stages with normal wall motion; circles, squares with abnormal wall motion.

degree in both normal and diseased ventricles. ${ }^{12-19}$ Our results accord with previous studies and show that LV cross sectional area change assessed by an ICE catheter in the RV can be related to $\mathrm{LV}$ stroke volume.

In the present study, alterations in regional wall motion were obtained by partially occluding the left anterior descending artery and increasing myocardial oxygen demand with dobutamine. Even in these ischaemic states, the linear relation between stroke area and stroke volume remained significant. In all of the dogs that we studied, ischaemia in the $\mathrm{LAD}$ territory resulted in a wall motion abnormality within the cross section image. If wall motion abnormalities were restricted to regions outside this imaging plane (such as the inferior base or the apex of the LV), then monitoring only the mid ventricular level could lead to an overestimation of LV function and a decreased sensitivity to detect ischaemia.

Although a significant relation between (stroke volume). This relation has previously been noted by transthoracic and transoesophageal echocardiography with correlations and errors in a similar range. ${ }^{78}$ These studies suggest that visualisation and measurement of the $\mathrm{LV}$ at this level can provide an estimate of overall LV function. Although the long axis dimension must be measured when absolute ventricular volume is examined, previous studies of ventricular dynamic geometry have demonstrated that $\mathrm{LV}$ ejection is accomplished primarily by inward motion along the short axis of the ventricle and that shortening in the long axis plane contributes to a lesser

stroke area and stroke volume was noted for each animal, there was significant variability in this relation from subject to subject. This variability may reflect the wide range of heart sizes that were studied (weights of the dogs ranged from 17 to $38 \mathrm{~kg}$ ). Other investigators, using 
transthoracic and transoesophageal echocardiography, also observed a similar degree of subject to subject variability in this relation..$^{7819}$ Thus although relative changes in $\mathrm{LV}$ function can be assessed within an individual subject, these values cannot be converted to an absolute stroke volume nor can direct comparisons between different individuals be made. The technique nevertheless can be used to fulfil its intended purpose: namely, monitoring of changes in ventricular function within a given individual.

Before the technique is applied clinically, several limitations must be considered. The accuracy of intracardiac ultrasound imaging will be influenced by the ultrasound transducer and the catheter placement. The 10 $\mathrm{MHz}$ transducer we used in this study has a reported penetration of about $6 \mathrm{~cm}$. In our experimental model, the endocardial borders of the LV were usually within $5 \mathrm{~cm}$ of the ICE catheter and thus it visualised them with minimal distortion. This degree of penetration is probably adequate for imaging the LV from the RV in small hearts such as those of infants, small children, and experimental preparations. A lower frequency transducer which provides greater beam penetration will be required for application in the adult human heart.

Catheter position and stability within the $\mathrm{RV}$ will influence the ability to obtain a true short axis image of the LV suitable for monitoring systolic function. Earlier studies showed that catheters positioned with a 30 degree deviation from the long axis of a circular vessel result in imaging planes which yield elliptical rather than circular cross sections of the vessel with up to a $20 \%$ overestimation of lumen area. $^{2021}$ In our study, using jugular venous access, we could always stabilise the catheter in an orientation parallel to the long axis of the LV to obtain representative cross sectional images of the LV. An ultrasound catheter with a steerable tip would further improve the ease of obtaining accurate short axis images of the LV. ${ }^{22}$ In addition, the catheter remained in a constant stable position with limited need for manipulation. This may be due, in part, to an anchoring effect of the heavily trabeculated right side of the septum. Both the optimal positioning and stability of the ICE catheter were possible with an internal jugular approach. Unfortunately, the current 10 French catheter size is impractical for routine clinical application.

The present device allows adequate imaging and measurement of left ventricular function in experimental preparations. With modifications to the current equipment, potential clinical applications include monitoring of $\mathrm{LV}$ function in patients in the intensive care unit or operating room and for improved assessment of myocardial contrast. With the integration of automatic border detection and this technique, continuous on-line monitoring of LV systolic function and pressure-area relations should be obtained. ${ }^{23} 24$

Intracardiac ultrasound imaging of the $\mathrm{LV}$ obtained by percutaneous introduction of the catheter into the RV provides adequate images of the LV cavity in this canine model. Detection of regional wall motion abnormalities are possible when they occur within the imaging plane. Measurements of the cross sectional area of the LV by this ICE approach correlate well with stroke volume, providing quantitative information which can be used during serial examinations to assess changes in LV systolic function. With the development of smaller catheters with lower frequency transducers to allow a greater penetration and with automated border detection this technique may be a valuable clinical method for continuous monitoring of $\mathrm{LV}$ function.

We thank Dr Bernard J Gersh for his helpful comments.

1 Fisher JP, Wolfberg CA, Mikan JS, Kiernan FJ, Fram DB, McKay RG, et al. Intracardiac ultrasound determination
of $\mathrm{LV}$ volumes: in vitro and in vivo validation. $\mathcal{f} \mathrm{Am}$ Coll of LV volumes: in vitro a

2 Chen C, Guerrero JL, Vazquez de Prada JA, Padial LR, Schwammenthal E, Chen MH, et al. Intracardiac ultrasound measurement of volume and ejection fraction in normal, infarcted, and aneurysmal left ventricles using a $10-\mathrm{MHz}$ ultrasound catheter. Circulation 1994;90: 1481-91.

3 Vazquez de Prada JA, Padial LR, Chen MH, Chen $M H$, Jiang $\mathrm{L}, \mathrm{He} \mathrm{J}$, et al. Assessment of right ventricular volume using a new $10 \mathrm{MHz}$ intracardiac ultrasound catheter: an in vitro validation study (abstract).
Circulation 1993;88:I-160.

4 Jiang L, de Prada JV, He J, Padial LR, Fallon JT, King ME, et al. Quantitative assessment of stenotic aortic valve area using intravascular echocardiography: in vitro validation (abstract). Circulation 1993;88:I-103.

5 Schwartz SL, Pandian NG, Hsu TL, Weintraub A, Cao QL. Intracardiac echocardiographic imaging of cardiac abnormalities, ischemic myocardial dysfunction, and myocardial perfusion: studies with a $10 \mathrm{MHz}$ ultrasound catheter. $\mathcal{F}$ Am Soc Echocardiogr 1993;6:345-55.

6 Schwartz SL, Pandian NG, Crowley R, Kumar R Intracardiac echocardiography without fluoroscopy: catheter. Am Heart $\mathcal{F}$ 1995;129:598-603.

7 Gorcsan J III, Gasior TA, Mandarino WA, Deneault LG, Hattler BG, Pinsky MR. On-line estimation of changes in left ventricular stroke volume by transesophageal echoleft ventricular stroke volume by transesophageal echo-
cardiographic automated border detection in patients undergoing coronary artery bypass grafting. Am $\mathcal{f}$ Cardiol 1993;72:721-7.

8 Gorscan J III, Lazar JM, Romand J, Pinsky MR. On-line estimation of stroke volume by means of echocardiographic automated border detection in the canine left ventricle. Am Heart f 1993;125:1316-23.

9 Bland JM, Altman DG. Statistical methods for assessing agreement between two methods of clinical measurement. Lancet 1986;i:307-10.

10 Tobis JM, Mallery J, Mahon D, Lehmann K, Zalesky P, Griffith $\mathrm{J}$, et al. Intravascular ultrasound imaging of human coronary arteries in vivo. Circulation 1991;83: 913-26.

11 Fitzgerald PJ, Ports TA, Yock PG. Contribution of localized calcium deposits to dissection after angioplasty: an observational study using intravascular ultrasound Circulation 1992;86:64-70.

12 Rushmer RF, Thal N. The mechanics of ventricular contraction: a cinefluorographic study. Circulation 1951;4: 219-28 13 Hawthorne E. Dynamic geometry of the left ventricle. $A m \mathcal{F}$
Cardiol 1966;18:566-73.

14 Bishop VS, Horwitz LD. Left ventricular transverse internal diameter: value in studying left ventricular function. Am Heart $\mathcal{F}$ 1970;80:507-14.

15 Leshin SJ, Mullins CB, Templeton GH, Mitchell JH. Dimensional analysis of ventricular function: effects of Dimensional analysis of ventricular function: effects of
anesthetics and thoracotomy. Am f Physiol 1972;222: 540-55.

16 Rankin JS, McHale PA, Arentzen CE, Ling D, Greenfield JC, Anderson RW. The three-dimensional dynamic geometry of the left ventricle in the concious dog. Circ Res 1976;39:304-13.

17 Lewis RP, Sandler H. Relationship between changes in left ventricular dimensions and the ejection fraction in man. Circulation 1971;44:548-57.

18 Walley KR, Grover RM, Raff GL, Benge W, Hannaford B, Glantz SA. Left ventricular dynamic geometry in the intact and open chest dog. Circ Res 1982;50:573-89.

19 Appleyard RF, Glantz SA. Two-dimensions describe left ventricular volume change during hemodynamic transients. Am f Physiol 1990;258:H277-84.

20 Chae JS, Brisken AF, Maurer G, Siegel R. Geometric accuracy of intravascular ultrasound imaging. $\mathcal{F} A m$ Soc
Echocardiogr 1993;6:158-65.

21 Mario CD, Madretsma S, Linker D. The angle of incidence of ultrasonic beam: a critical factor for image quality in intravascular ultrasonography. Am Heart $\mathcal{F} 1993$; 
22 Tardif JC, Cao QL, Schwartz S, Vannan M, Pandian N. Intracardiac echocardiography with a steerable linear array device permits high resolution imaging of cardiac chambers and valves with an extended depth of field and chambers and valves with an extended depth of field and
thus left heart imaging from the right side. $\mathcal{F} \mathrm{Am}$ Coll Cardiol 1994;1A:484A.

23 Gorcsan J III, Romand JA, Mandarino WA, Deneault LG Pinsky MR. Assessment of left ventricular performance by on-line pressure-area relations using echocardio- graphic automated border detection. $7 \mathrm{Am}$ Coll Cardiol 994;23:242-52.

24 Katz WE, Murali S, Mahler C, Mandarino WA, Kormos RL, Gorcsan J III. Automated noninvasive estimation of left ventricular contractility using end-systolic pressurearea relations from echocardiographic acoustic quantification and peripheral arterial pressure (abstract). $\mathcal{F} \mathrm{Am}$ Coll Cardiol 1994;90:I-495.

\title{
SHORT CASES IN CARDIOLOGY
}

\section{Haemorrhagic bullae in a patient with lichen sclerosus et atrophicus treated with streptokinase}

\author{
Heather M Dunn, Raymond A Fulton
}

\section{Altnagelvin Area \\ Hospital, \\ Londonderry, \\ Northern Ireland \\ H M Dunn \\ R A Fulton \\ Correspondence to: Dr H M Dunn, Cardiac Unit, Altnagelvin Area Hospital, Glenshane Road, Londonderry BT47 1SB Northern Ireland. \\ Accepted for publication 30 April 1996}

A 62 year old woman with no previous history of cardiac disease was admitted with a 2 h 30 min history of chest pain. The electrocardiograph showed ST changes in the inferolateral leads in keeping with a diagnosis of acute myocardial infarction. She had no recognised contra-indications to thrombolytic therapy.

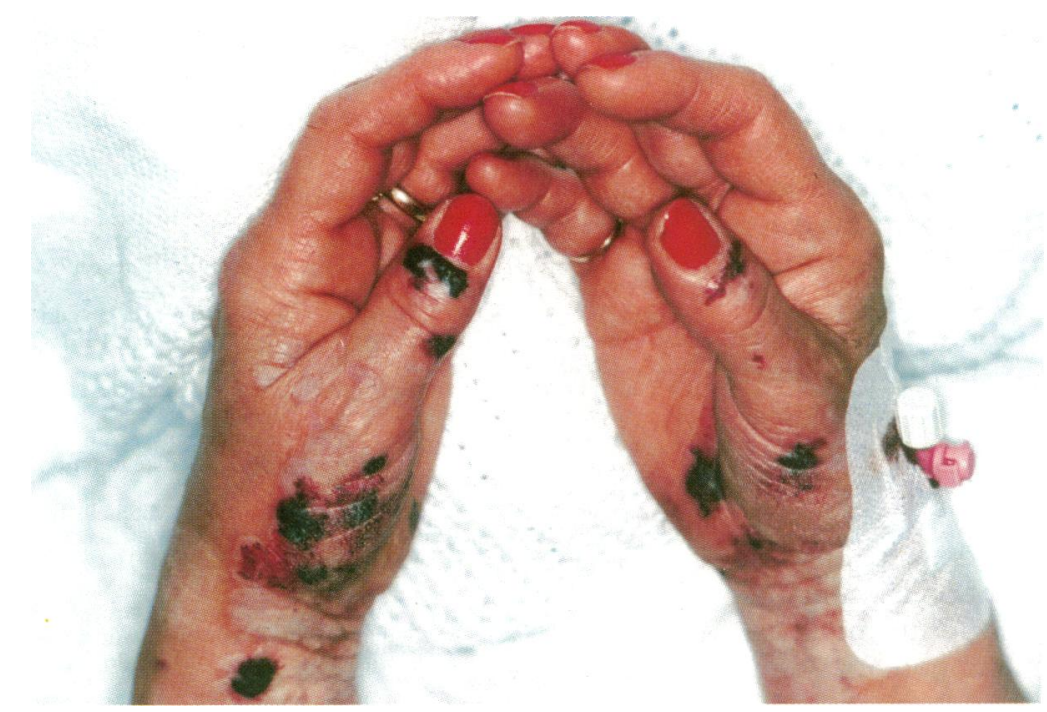

Haemorrhagic bullae in a patient with lichen sclerosus et atrophicus treated with streptokinase
She was treated with soluble aspirin $(150 \mathrm{mg})$ and streptokinase ( 1.5 million units) followed by heparin infusion (1000 units per hour).

On the day after admission the patient complained of pain and swelling on the flexor surfaces of both wrists and in the periumbilical region. On examination there were atrophic plaques on the wrists, umbilical region, and vulva. Haemorrhage had occurred into these plaques with the formation of haemorrhagic bullae (figure). This significantly limited movement at the wrists. On questioning the patient gave a long history of white patches affecting the flexor surfaces of the wrists, the vulva, and periumbilical region. A diagnosis of lichen sclerosus et atrophicus had been made and the patient had previously noted minor bullae related to trauma. The heparin was stopped and the patient was treated with simple analgesia.

Lichen sclerosus is an uncommon disease of unknown aetiology presenting as small porcelain-like shiny round macules which usually aggregate into atrophic plaques. Occasionally bullae, telangiectasia, and purpura may spontaneously occur in the plaques. Treatment with streptokinase in this case caused the sudden formation of painful haemorrhagic bullae in the pre-existing lichen sclerosus. 